pag

Business School

WORKING PAPER SERIES

Working Paper

2014-323
Pouvoir et leadership dans les canaux de distribution : la logistique face aux jeux des acteurs

Virginie Noireaux

Carole Poirel

http://www.ipag.fr/fr/accueil/la-recherche/publications-WP.html

IPAG Business School

184, Boulevard Saint-Germain

75006 Paris

France 


\title{
Pouvoir et leadership dans les canaux de distribution : la logistique face aux jeux des acteurs
}

\author{
Virginie Noireaux \\ Carole Poirel
}

\section{Résumé}

Le pouvoir est une notion clef de l'analyse des canaux de distribution. Or, plusieurs chercheurs du CRET-LOG l'ont mobilisé pour appréhender les jeux d'acteurs et en particulier les relations logistiques entre industrie et commerce. Cet article se propose de rendre compte de leurs travaux concernant l'opérationnalisation des sources de pouvoir, les différents types de leadership et les modalités stratégiques de résistance.

\begin{abstract}
Power is a key concept when analysing the distribution channels. Several CRET-LOG members have used it in order to understand the interplay of actors and the logistics relationships between industry and retailing. This article presents their works on the understanding and implementation of the power sources, the exercise of power, and the strategy of resistance.
\end{abstract}

Post Print : Management \& Avenir, 2009/4, n²4.

our acheminer des biens du producteur au consommateur, une séquence d'institutions est nécessaire, au sein de laquelle se posent notamment des questions de coûts, de croissance et de stratégies. Comment industriels et distributeurs font-ils pour aborder et régler ensemble ces questions et faire fonctionner le canal de distribution dans les meilleures conditions possibles ? Car, on perçoit bien que si le canal oblige tout un chacun à un minimum de coopération sinon de coordination, il n'annihile pas pour autant - et peut-être même les exacerbe-t-il ? - tous les enjeux de pouvoir et de leadership. Qui fixe les règles ? Comment ? Selon quelle légitimité ? Avec quelles limites? Au printemps 2008, avant que la crise bancaire éclate et que la crise économique se généralise au monde occidental, la baisse du pouvoir d'achat était déjà un sujet de débat auquel le 
canal n'était guère étranger. Les questions portaient alors sur la hausse des prix et les acteurs susceptibles d'en être à l'origine. Ces questions n'ont pas manqué de rappeler les responsabilités de ceux qui exercent le pouvoir dans les canaux de distribution. Or, d'après un sondage effectué en mai 2008, les français étaient plutôt partagés : si 40\% d'entre eux attribuaient la responsabilité de la hausse des prix aux grandes surfaces, $44 \%$ considéraient les industriels comme les plus fautifs.

Comment définir le pouvoir ? C'est en effet un concept polymorphe que les théoriciens du canal de distribution ont commencé à mobiliser dès la fin des années 1960 (Stern, 1969) pour expliquer que les comportements d'acteurs ne dépendaient pas seulement de la variable économique de coût. Il est fréquemment fait appel à la définition de Dahl (1971) pour qui le pouvoir d'un acteur A sur un acteur B n'est autre que la capacité de A d'amener B à faire quelque chose qu'il n'aurait pas fait autrement. La relation entre A et B est donc, inégale mais cependant réciproque (March, 1955 ; Crozier et Friedberg, 1977) : tous les acteurs ont du pouvoir mais certains en ont plus que d'autres ! El-Ansary et Stern (1972) ont été parmi les premiers à appliquer le concept au canal, indiquant que le pouvoir de A est sa capacité à maîtriser les variables de décisions relatives à la stratégie marketing de $\mathrm{B}$, situé à un niveau du canal différent du sien. Le pouvoir prend naturellement diverses formes selon les situations. Il s'apparente à la domination quand $\mathrm{A}$ fait appel à la contrainte pour imposer sa volonté à $\mathrm{B}$. Il peut être aussi autorité lorsque B s'incline par crainte ou par respect, sans que A ait recours à la force. Rappelons que pour Weber (1971), avoir de l'autorité, c'est faire observer volontairement ses ordres en s'appuyant sur les règles de droit, les traditions ou des qualités charismatiques. Il y a également l'influence, qui se présente comme un pouvoir non hiérarchique de $\mathrm{A}$, fondé sur la persuasion et la séduction, voire la manipulation, mais tout autant capable d'entraîner un changement d'attitudes, d'opinions ou de façon d'agir chez B. Comment différencier maintenant pouvoir et leadership ? Pour nous, le leadership est un anglicisme synonyme d'exercice du pouvoir qu'il s'agisse de domination, d'autorité ou d'influence. Dans le même ordre d'idées, le leader est le détenteur d'un avantage de pouvoir.

Cet article se propose de rendre compte de différentes contributions de chercheurs du CRETLOG, autour de trois principales problématiques qui seront successivement développées par la suite. Une première problématique est relative aux sources de pouvoir. C'est effectivement en identifiant les sources qu'il est possible de repérer celui qui exerce le pouvoir et celui qui le subit, mais cette identification du leader pose des problèmes méthodologiques délicats. Bonet (1999) et Poirel (2003) ont tenté de les dépasser en recherchant des mesures appropriées : la première en s'appuyant sur les travaux de French et Raven (1959), la deuxième en utilisant les 
apports de la stratégie d'entreprise et plus particulièrement le concept de pouvoir de négociation développé par Porter $(1982,1986)$.

Une deuxième problématique considère le mode d'exercice du pouvoir et ses répercussions sur le degré de satisfaction des membres vis-à-vis du leader ainsi que sur le climat général du canal plus ou moins favorable aux transactions et au développement de la coopération (Mallen, 1964 ; Schul, Pride et Little, 1983). Alors que la plupart des recherches se focalisent sur les aspects marketing, c'est à la dimension logistique que s'est consacré Paché seul (1999, 2002) ou en collaboration (Filser et Paché, 2008) dans plusieurs articles et ouvrages.

En abordant la logistique dans une perspective sociopolitique à travers le jeu des acteurs, cet auteur offre ainsi une alternative à l'approche économique, traditionnelle et rationaliste, dont la recherche opérationnelle constitue la figure de proue. Egalement affiliée au CRET-LOG, Noireaux (2006) a montré combien il était important pour le canal, que le leader soit reconnu et légitimé dans ses fonctions par ses coéchangistes. La gestion des déchets industriels qu'elle a choisi d'étudier en tant que canal de distribution inversée, requiert de la part des leaders (en l'occurrence, les groupes d'élimination) une certaine habileté dans l'exercice de leur pouvoir ainsi qu'un certain degré d'innovation organisationnelle, pour que les producteurs cessent d'adopter des comportements d'évitement et qu'ils adhèrent au projet écologique.

Une troisième et dernière problématique concerne la portée du pouvoir et la façon dont ceux qui le subissent, y réagissent. Sont-ils dans l'acceptation et la soumission ou bien dans le refus et la résistance ? Ces questions, qui ne sont pas neuves au demeurant, laissent entendre que le pouvoir n'est pas une structure relationnelle figée mais bien au contraire, capable d'évoluer et de faire évoluer avec elle, le canal dans son entier. Cette dynamique du pouvoir est soustendue par des stratégies d'acteurs que Poirel (2003, 2008) s'est proposé d'analyser dans le cadre de la distribution du livre en France. Les résultats de ses recherches ont montré que les stratégies de résistance n'étaient pas réservées aux acteurs du commerce les plus importants en termes économiques mais que d'autres intervenants, de taille modeste mais porteur d'un projet comportant une dimension culturelle affirmée, trouvaient leurs propres modalités stratégiques pour résister au pouvoir de leurs fournisseurs. 


\section{1 - Les origines du pouvoir ou comment mesurer le pouvoir?}

S'interroger sur les origines est une question importante qui permet de comprendre les ressorts du pouvoir mais également de localiser les leaders dans le canal. Pourtant, cette question se heurte à un problème méthodologique lié à la mesure du pouvoir. En effet, de nombreuses recherches consacrées au canal de distribution ont montré que le pouvoir de A sur B était perçu par B quand il reposait sur la sanction. En revanche, il n'est pas perçu par B quand il s'appuie sur l'expertise (Hunt et Nevin, 1974 ; Etgar, 1978 ; Lusch et Brown, 1982). Pour Lusch et Brown (1982), ce paradoxe tient au fait que l'emploi de sources non coercitives comme l'expertise jouent dans le sens de l'adhésion de B aux normes et valeurs de A, le processus de socialisation à l'œuvre donnant à $\mathrm{B}$ le sentiment d'agir en toute indépendance, sans avoir conscience du pouvoir qu'il subit. Si cette explication est convaincante, les résultats demeurent : le pouvoir perçu est différent du pouvoir réel. Autrement dit, A dispose d'un pouvoir sur B quand B pense que A exerce effectivement un pouvoir sur lui, et ce, même indépendamment des sources et des instruments inhérents au pouvoir de A (March, 1955). En d'autres termes, un acteur considéré comme puissant est puissant quelles que soient ses sources réelles de pouvoir. Dans ces conditions, ne faudrait-il pas considérer la perception du pouvoir comme une nouvelle source de pouvoir? Suivant Filser (1989), les problèmes de mesure du pouvoir sont tels qu'il faut en définitive accepter d'évaluer un pouvoir perçu et non pas un pouvoir réel ou bien avoir conscience d'apprécier non pas le pouvoir en lui-même mais ses sources.

Connaissant ces limites, des chercheurs du CRET-LOG ont proposé deux méthodes différentes mais complémentaires pour opérationnaliser les sources de pouvoir. Bonet (1999) d'une part, a proposé une analyse du discours des acteurs comme révélateur de leurs comportements stratégiques et Poirel (2003) d'autre part, une grille d'analyse des relations de pouvoir entre clients et fournisseurs issue de la stratégie d'entreprise.

\section{1 - L'analyse propositionnelle du discours des acteurs}

Aux cinq sources de pouvoir identifiées par French et Raven (1959), à savoir la sanction, la récompense, l'expertise et la valeur de référence et d'identification, il convient d'en ajouter une sixième : la détention d'informations (Raven et Kruglanski, 1970 ; Crozier et Friedberg, 
1977). Pour les évaluer, Bonet (2000) propose de recourir aux méthodes déclaratives. Des entretiens semi directifs conduits auprès de responsables d'entreprises commerciales et industrielles permettent de recueillir des données discursives sur le thème des relations clients-fournisseurs. L'innovation méthodologique réside dans l'emploi de l'analyse propositionnelle du discours conjugué à l'usage, habituel en Gestion, de l'analyse de contenu thématique. Les déclarations intégralement retranscrites sont ainsi analysées au double plan $\mathrm{du}$ fond (analyse de contenu) et de la forme (analyse de discours). Pour l'auteur, les deux méthodes se complètent, l'analyse de contenu organisant le fond du discours selon une vision thématique avec une certaine subjectivité, l'analyse de discours découpant mécaniquement le texte pour en présenter les éléments de forme dans une démarche plus objective.

L'auteur a réalisé l'analyse propositionnelle du discours au moyen du logiciel Tropes développé au début des années 1990 par deux chercheurs en psychologie sociale de l'Université Paris VIII : Landré et Molette. Ces derniers avaient pour objectif de fournir un outil d'analyse mettant en évidence les sens cachés des textes et des discours (notamment politiques) par une analyse lexicale fine dont l'unité d'enregistrement est la proposition au sens grammatical du terme. Il s'agit de rendre visible des éléments discursifs cachés à la seule lecture des retranscriptions. La masse importante de données lexicales traitées par le logiciel présente en outre, l'avantage d'être synthétisée grâce à l'analyse factorielle des correspondances sous la forme d'un ensemble « d'univers représentationnels » opposant, dans le cadre du travail de Bonet (1999), le conflit et la coopération dans le canal de distribution. L'un des principaux résultats de ce travail a été de montrer que la logistique n'était pas un univers aussi coopératif entre membres du canal qu'elle le laissait espérer. Alors que des rapprochements ont lieu entre industriels et distributeurs pour coordonner les opérations au moyen d'outils tels que la gestion partagée des approvisionnements (GPA) ou la réponse optimale au consommateur (ou Efficient Consumer Response), des conflits de nature stratégique, ceux-là, surgissent. Ils sont liés au partage des coûts et des bénéfices inhérents à la gestion des opérations logistiques ainsi qu'à la maîtrise de l'information nécessaire au pilotage des flux. La logistique présente ainsi une certaine ambivalence, étant tout à la fois porteuse de collaborations et de désaccords profonds. 


\section{2 - Les apports de la stratégie à la mesure des sources de pouvoir}

Les déterminants du pouvoir de négociation que Porter $(1982,1986)$ a identifiés, constituent autant de sources de pouvoir. Certaines de ces sources, comme le différentiel de concentration, ne nécessitent pas pour être évaluées de mesures déclaratives car des statistiques sectorielles permettent de les apprécier. Quand des entretiens s'avèrent nécessaires pour d'autres types de sources, ils concernent avant tout des professionnels ou des observateurs avertis, choisis à titre d'experts et non en tant que participants. Ceux-ci sont interrogés de surcroît sur des domaines relativement objectifs concernant les produits (degré de différenciation, existence de substituts, etc.) ou les relations verticales (coûts de transfert, qualité liée, etc.). Des données secondaires issues de la presse professionnelle, de rapports publics ou de documents internes aux organisations, peuvent par ailleurs venir s'ajouter. L'ensemble des éléments ainsi collectés est ensuite interprété à travers le prisme portérien. Les sources de pouvoir du client sont identifiées puis celles du fournisseur, avant d'être confrontées les unes aux autres pour faire émerger une configuration relationnelle comme Cox (2001) l'a suggéré (voir Figure 1).

Figure 1 - La matrice de pouvoir selon Cox (2001, p.13)

\begin{tabular}{c}
$\begin{array}{c}\text { Attributs du } \\
\text { pouvoir du } \\
\text { client (par } \\
\text { rapport au } \\
\text { fournisseur) }\end{array}$ \\
\cline { 2 - 3 }
\end{tabular}

En situation d'interdépendance, le client et le fournisseur disposent tous deux de sources de pouvoir importantes. La relation est en ce cas relativement équilibrée. Dans le cas de l'indépendance, aucune source de pouvoir n'apparaît significativement forte chez le fournisseur ou le client, ce qui veut dire que les interactions restent limitées entre eux. Quand l'un domine l'autre, en revanche, c'est qu'il dispose de sources de pouvoir plus nombreuses et/ou plus robustes que son coéchangiste. Surviennent dans ces conditions des déséquilibres patents plus ou moins prononcés selon les cas.

Dans le cas de la distribution du livre en France, qui a constitué un terrain d'investigation pour Poirel (2003), c'est la domination du fournisseur qui est ressorti comme la configuration 
relationnelle prédominante. En effet, de façon résumée, l'édition dispose vis-à-vis du commerce du livre, de trois grandes sources de pouvoir :

- Une source économique provenant des écarts de concentration entre secteurs ainsi que des différences de taille entre intervenants ;

- Une source culturelle inhérente aux caractéristiques du produit-livre, ce dernier étant par nature fortement différencié et peu substituable ;

- Une source organisationnelle liée à la maîtrise de la gestion commerciale et logistique du canal par les principaux groupes d'édition.

Mais il convient d'apporter deux précisions à ces résultats. D'une part, ce n'est pas l'éditeur qui détient l'avantage de pouvoir mais quelques groupes d'édition qui ont intégré les fonctions d'intermédiation commerciale et de logistique au sein du canal de distribution. D'autre part, si le commerce du livre est globalement moins bien positionné que l'édition dans la négociation verticale, quelques grandes enseignes ont les moyens d'exercer un réel contrepouvoir vis-à-vis de l'ensemble de la sphère éditoriale. Ce point sera repris dans la troisième partie consacrée à la portée du pouvoir.

\section{2 - L'exercice du pouvoir ou comment le leader peut-il utiliser la logistique pour régner sur le canal ?}

Les sources de pouvoir permettent au leader d'influencer les objectifs et les stratégies des acteurs du canal et d'en contrôler les différents aspects opérationnels (Stern et El-Ansary, 1988). Le leader cherche à imposer le niveau de service qui permet de répartir les activités entre les différentes institutions (Filser, 1989, 2000). Le rôle du leader soulève des questions d'opérationnalité, notamment quant à son intensité et quant à sa forme. L'abus ou l'utilisation trop discrète du pouvoir sont néfastes aux canaux. En effet, son usage imprudent conduit à la diminution de l'efficience dans le canal, voire à la destruction de celui-ci (Stern et Heskett, 1969 ; Wilkinson, 1979). Inversement, un trop faible recours au pouvoir tend à augmenter la dépendance des membres vis-à-vis des marchés intermédiaires et à fragiliser le canal (Etgar, 1977). Cette constatation a conduit les auteurs à s'intéresser aux attitudes attendues du leader. 
Pour Stern et El-Ansary (1988), une des clés principales du contrôle du canal est la réduction des conflits, ce qui dépend du style de leadership employé. Plusieurs travaux montrent qu'un haut niveau de satisfaction des membres du canal engendre un meilleur moral, une meilleure coopération, des ruptures de relations moins fréquentes et une réduction des litiges (Dwyer, 1980 ; Anderson et Narus, 1984 ; Stern et El-Ansary, 1988). Pour atteindre cet objectif, le leader peut exercer son pouvoir de différentes manières (Mallen, 1964 ; Schul, Pride et Little, 1983). La première correspond à un style directif, proche de la domination évoquée en introduction. Le leader organise, définit les tâches et évalue les performances de manière unilatérale. La seconde est plus participative. Elle s'apparente davantage à l'autorité au sens de Weber (1971) et à la persuasion. Le leader consulte les autres membres de manière à décider collectivement de la répartition des opérations et de leur coordination. Schul, Pride et Little (1983) ajoutent une forme intermédiaire, où le leader considère les besoins et les émotions des autres membres dans ses décisions pour créer un environnement plus convivial et plus agréable. Dans cette optique, les chercheurs du CRET-LOG ont cherché à approfondir cette notion d'exercice du pouvoir. Ils ont pour cela exploré deux voies particulières. La première considère la logistique comme un moyen d'exercer le pouvoir (Paché, 1994, 2002 ; Filser et Paché, 2008). La seconde avance la notion de reconnaissance du leader pour améliorer le climat au sein du canal (Noireaux, 2006).

\section{1 - La logistique comme moyen d'exercer le leadership}

Dans le secteur de la distribution, si les formats de vente n'ont pas connu d'évolution majeure depuis une dizaine d'années, toutes les opérations amont ont subi de profonds changements liés à trois tendances lourdes. La première concerne la concentration des détaillants, qui inverse durablement les rapports de force entre commerçants et industriels. La deuxième fait référence à l'internationalisation des réseaux de vente et à la mondialisation des sources d'approvisionnement. Enfin, la troisième recherche les gains de productivité dans le canal pour maintenir les marges des détaillants. Ces évolutions placent les détaillants au centre du canal. C'est à eux que revient aujourd'hui le leadership.

Paché (2002) puis Filser et Paché (2008) proposent d'intégrer la dimension logistique à l'exercice du pouvoir afin de renouveler l'approche «instrumentale » qui découle de la distinction entre les sources de pouvoir non coercitives et coercitives proposée par Hunt et Nevin (1974). Pour eux, alors que la majorité des travaux académiques privilégient les variables d'action marketing pour mettre en œuvre les sources non coercitives, il est légitime 
d'y intégrer aujourd'hui la gestion des opérations logistiques. Ils détaillent cette relation pour chaque source non coercitive du pouvoir (voir Tableau 1).

Tableau 1 - Gestion des opérations logistiques dans l'exercice des sources non coercitives du pouvoir (Source : d'après Filser et Paché, 2008)

\begin{tabular}{|l|l}
\hline Sources non coercitives & Rôle et effets des opérations logistiques \\
\hline La récompense & $\begin{array}{l}\text { Remplacement des livraisons directes } \\
\text { l'enlèvement usine ou par la division des } \\
\text { travers les entrepôts ou plates-formes. Pe। } \\
\text { gains de productivité et/ou un « recentras } \\
\text { créatrices de valeur. }\end{array}$ \\
\hline L'expertise & $\begin{array}{l}\text { Compétences logistiques des détaillants pe } \\
\text { au bon endroit, au bon moment et en quan } \\
\text { à l'industriel les ruptures en rayon ce qui lir } \\
\text { de marques de la part des consommateurs }\end{array}$ \\
\hline La légitimité & $\begin{array}{l}\text { Découle de l'expertise du pilotage des flux. } \\
\text { une certaine « autorité de type charismatiq! }\end{array}$ \\
\hline La valeur de référence & $\begin{array}{l}\text { Adhésion au système logistique du dé } \\
\text { industriels (notamment les PME région } \\
\text { visibilité par le consommateur final, un a } \\
\text { capitaux pour financer leur croissance. }\end{array}$ \\
\hline
\end{tabular}

En ce qui concerne les sources coercitives, la logistique représente incontestablement une autre facette du pouvoir du détaillant. Ce dernier définit des sanctions telles que des pénalités financières plus ou moins élevées, lorsque son cahier des charges n'est pas suivi par l'industriel. Son objectif est de modifier le comportement de l'industriel défaillant eu égard à la qualité de service qu'il a fixée. Cependant, Filser et Paché (2008) remarquent que l'utilisation de la logistique comme source coercitive du pouvoir doit être relativisée pour deux raisons : premièrement, parce que les sanctions éventuelles s'appliquent dans la phase de référencement; deuxièmement, parce que les dysfonctionnements repérés a posteriori ne conduisent généralement pas au déréférencement des fournisseurs. 
Les auteurs insistent sur le fait que les sources non coercitives du pouvoir demeurent le plus souvent tolérables pour les industriels, alors que la sanction entraine des stratégies qui alternent entre évitement, affrontement et tentatives de contre-pouvoir. Ils présentent alors deux types de démarches collaboratives soutenues par les industriels pour limiter le pouvoir des distributeurs : la gestion partagée des approvisionnements (GPA) et le «collaborative planning, forecasting and replenishment »(CPFR). Tous deux permettent aux industriels de se réapproprier l'expertise de la gestion des stocks et l'organisation des livraisons dans les magasins par une meilleure connaissance de la demande. Ils concluent que la mise en œuvre de ces démarches reste délicate du fait de la perte d'autonomie partielle des acteurs sur le plan décisionnel et de l'affaiblissement de leur territoire d'expertise.

\section{2 - Vers la reconnaissance du leadership dans les organisations logistiques}

Le second axe de recherche s'intéressant à l'exercice du leadership, étudie ces démarches collaboratives dans un cas particulier du canal de distribution: le canal de distribution inversée (Stern et El-Ansary, 1988). Ce dernier représente «l'ensemble des membres qui prennent possession ou facilitent l'échange d'un bien ou d'un matériau considéré comme déchet par son utilisateur dans le but de le réutiliser, le recycler ou le stocker» (Noireaux, 2006, p.48).

Hormis le fait que ce canal présente des particularités techniques, technologiques ainsi qu'au plan des compétences, il s'avère particulièrement intéressant pour analyser les stratégies d'acteurs. En effet, le contexte est proche de celui de la distribution (voir tableau 2) à une différence près. Dans la distribution, le manque d'innovation en matière de format de vente limite les producteurs dans la mise en place de stratégies, notamment collaboratives face aux distributeurs-leaders (Filser et Paché, 2008). Les canaux sont limités dans leurs nombres et dans leurs formes, ce qui astreint les producteurs à subir le pouvoir du leader pour distribuer leurs produits. Dans la distribution inversée, l'utilisation du pouvoir inconsidérée par les groupes d'élimination de déchets (ici, les leaders) est sanctionnée par l'évitement du canal «réglementaire », au profit de canaux illégitimes comme les décharges sauvages (Noireaux, 2008). 
Tableau 2 - Caractéristiques du canal de distribution et du canal de distribution inversée (Sources : d'après Noireaux, 2006 et Filser et Paché, 2008)

\begin{tabular}{|l|l|l|}
\hline Caractéristiques & Distribution & Distribution inversée \\
\hline \multirow{5}{*}{ Canaux } & $\begin{array}{l}\text { Producteurs de toutes tailles (Gros } \\
\text { industriels et PME) }\end{array}$ & $\begin{array}{l}\text { Producteurs de toutes tailles } \\
\text { industriels et PME) }\end{array}$ \\
\cline { 2 - 4 } & Concentration des distributeurs & $\begin{array}{l}\text { Concentration des } \\
\text { d'élimination }\end{array}$ \\
\cline { 2 - 3 } Filières & $\begin{array}{l}\text { Intégration des aspects logistiques } \\
\text { par les distributeurs }\end{array}$ & $\begin{array}{l}\text { Intégration des aspects logi } \\
\text { par les groupes d'élimination }\end{array}$ \\
\hline & $\begin{array}{l}\text { Adhésion à un canal de distribution } \\
\text { pour assurer la survie des } \\
\text { industriels (flux stratégiques) }\end{array}$ & $\begin{array}{l}\text { Adhésion à un canal, légal ou } \\
\text { ne mettant pas en péril la su } \\
\text { l'entreprise (flux non-stratégiq }\end{array}$ \\
\hline
\end{tabular}

L'évitement a un impact direct sur la notion de leadership dans le canal et met en exergue la notion de leader reconnu. En effet, dans la distribution, le flux est en relation directe avec le cœur de métier et donc la survie des producteurs, ce qui peut les pousser à accepter l'exercice d'un pouvoir élevé par un leader pourtant en mal de reconnaissance. La concentration des distributeurs et le manque d'innovation en matière de formats de magasins accentuent ce phénomène. La gestion des flux est dans ce cas hautement stratégique. A contrario, dans les canaux de distribution inversée, la notion d'évitement est prégnante. Le leader doit donc être reconnu par les autres membres pour que la survie du canal soit assurée. La dimension stratégique inhérente à la gestion des flux est généralement perçue comme moins fondamentale par les acteurs, sauf lorsqu'ils ont fait de leur « image verte » un élément clef de leur stratégie générale. Ceuxlà acceptent alors le pouvoir des groupes d'élimination car ils dépendent d'eux pour mettre en œuvre une partie de leur stratégie mais ne les reconnaissent pas pourtant comme leaders. Néanmoins, la reconnaissance du leader dans les canaux de distribution (inversée ou non) permet à la fois d'améliorer le climat au sein du canal et d'assurer à long terme sa survie. En effet, quelles seraient les réactions des producteurs en cas d'innovation majeure en termes de formats de magasins (donc d'une nouvelle concurrence dans les canaux) ? Elle pourrait remettre en question la survie des canaux actuels considérés comme étant dominés de manière trop directive par les distributeurs-leaders.

La reconnaissance du leader se mesure à partir de trois variables (Noireaux, 2006 et 2008) : l'adhésion des membres à sa vision, les compétences et la légitimité. Alors que le leader non reconnu utilise uniquement l'ensemble de ses sources de pouvoir pour exercer son contrôle, le leader reconnu élabore et met en œuvre une vision, qui lui permet d'être accepté par les autres 
membres, d'augmenter leur satisfaction et de diminuer les comportements d'évitement. Ainsi, la concentration des sources de pouvoir ne suffit-elle pas. Il faut encore que les autres membres adhèrent symboliquement à l'organisation et aux objectifs que le leader met en place.

L'importance des comportements d'évitement dans le canal de distribution inversée (ADEME, 2004) incite les groupes d'élimination à innover dans l'exercice de leur pouvoir. Une de ces innovations (Noireaux, 2006), consiste en la création d'une coalition de parties prenantes pour diriger le canal (cf. Encadré 1). Cependant, le processus de mise en œuvre d'une telle stratégie est difficile et délicat. Le modèle processuel de l'émergence de la coalition de parties prenantes comme leader reconnu (Noireaux, 2006) souligne la longueur du processus, l'importance des stratégies d'acteurs, de la communication et des choix logistiques.

\section{Encadré 1 : La coalition de parties prenantes, une innovation dans l'exercice du pouvoir} La coalition de parties prenantes dans le canal de distribution inversée est composée d'acteurs privés et publics (ADEME, producteurs, agences de l'eau, collectivités territoriales, groupes d'élimination...), qui collaborent à la formation des objectifs du canal. Les groupes d'élimination restent alors leaders, du fait qu'ils possèdent la majorité des sources de pouvoir dans le canal. Par contre, l'exercice du pouvoir passe d'un style directif à un style participatif, ce qui a pour effet, dans ce contexte, une reconnaissance du leadership. Ces organisations ont été amorcées par les industriels pour contrecarrer un trop grand pouvoir perçu des groupes d'élimination. Or, aujourd'hui, ces groupes se sont appropriés l'initiative de ces démarches car elles leur permettent d'accroître leur activité en limitant les comportements d'évitement des producteurs.

\section{3 - La portée du pouvoir : la logistique pour apprendre à résister au leader}

Le pouvoir ne s'exerce pas sans résistance. C'est un point sur lequel s'accordent de nombreux spécialistes du pouvoir issus de disciplines variées (Sciences politiques, Economie, Théorie des organisations, Sociologie des organisations, Stratégie etc.). Pour Baechler (1978) par exemple, «la volonté de A rencontre la volonté soumise de B. Mais si cette volonté est soumise par nature, sans possibilité aucune de jamais désobéir, il n'y aurait pas de pouvoir 
non plus. Le pouvoir réside donc dans la capacité de dominer une volonté possiblement récalcitrante. » Un auteur plus fréquemment cité dans les recherches sur le canal de distribution, Emerson (1962), définit le pouvoir de A comme «la quantité de résistance de B surmontée par $\mathrm{A} »$. La résistance saisit l'exercice du pouvoir à travers le jeu des influences réciproques entre acteurs. Elle met en exergue le caractère dynamique des relations qui se tissent entre les membres du canal, révélant les forces contraires au leader qui se voit ainsi contesté, limité et contrôlé dans l'exercice de son pouvoir.

Certes, la notion n'est pas nouvelle mais elle connaît depuis plusieurs années une nouvelle vigueur dans les Sciences Humaines et Sociales, avec la montée de ce qui a été convenu d'appeler l'altermondialisme. En Sciences de Gestion, des auteurs se sont emparés d'elle pour redécouvrir des phénomènes stratégiques et organisationnels comme en témoigne une table ronde organisée sur le sujet lors de la dernière conférence de l'AIMS (Courpasson et Golsorkhi, 2008). Concernant le canal de distribution plus précisément, les vieilles théories béhavioristes constituées dans les années 1970 autour des concepts de conflit, de dépendance et de contrepouvoir, ont été rafraîchies par un courant stratégique du canal qui a émergé au début des années 1990 avec les travaux de Lapassouse $(1989,1991)$ et de Pras (1991). Ces deux auteurs s'appuient sur la notion de résistance pour aborder les stratégies d'acteurs dans le canal: le premier en appliquant la théorie de la chaîne de valeur de Porter (1986) à l'analyse de la stratégie du distributeur en milieu industriel, le second en s'intéressant aux différents registres stratégiques (affrontement, évitement et coopération) et à leurs interactions au sein du canal.

Dans le cadre du CRET-LOG, Poirel (2003) a souhaité reprendre et prolonger ces travaux en les appliquant à un secteur, la distribution du livre, où des effets de domination s'expriment de façon assez nette (voir §1.2.). En effet, alors que Lapassouse (1989) n'a pas pu prouver l'existence de stratégies de résistance dans le secteur qu'elle avait choisi, Pras (1991) a essentiellement fourni un cadre conceptuel. Il était donc pertinent de tenter à nouveau une investigation empirique. Or, deux résultats importants sont apparus à la suite des recherches de Poirel $(2003,2008)$. Le premier a révélé que les stratégies de résistance n'émergeaient pas toutes dans les mêmes conditions et que si la taille comptait, d'autres conditions plus qualitatives amenaient des acteurs de taille modeste à mobiliser eux aussi des moyens pour résister. Le deuxième a porté plus particulièrement sur le rôle de la logistique comme moyen pour les acteurs d'apprendre à s'opposer à leurs fournisseurs dans le canal. 


\section{1 - Les stratégies de résistance : entre marketing et logistique}

La stratégie de résistance se définit comme la capacité d'un membre à garder la maîtrise de ses variables de décision stratégique qu'un autre cherche à influencer (Poirel, 2003). Avec une telle stratégie, les acteurs vont souvent au-delà d'une simple protection de leur champ d'actions, car en retrouvant plus de marges de manœuvre, ils deviennent aussi plus influents. En effet, celui qui résiste, se place avant tout dans une logique d'affrontement, soit généralisée en touchant tous les territoires possibles de la relation soit localisée en privilégiant quelques domaines jugés plus sensibles. Cependant, la résistance n'exclut ni la coopération (où l'acteur obéit loyalement et cherche à renforcer les liens) ni l'évitement (où il se désengage de la relation et limite les contacts avec le leader). Les protagonistes peuvent en effet, faire se succéder dans le temps les différents registres (résistance puis coopération, par exemple) ou bien les mobiliser simultanément dans différents domaines (notamment, la coopération dans la logistique et l'affrontement dans le domaine commercial).

Les quatre stratégies de résistance que Poirel (2003) a pu mettre en exergue à la suite d'une étude quantitative menée auprès de 97 représentants du monde de la librairie française (indépendants, groupements et chaînes), se situent à la fois dans le canal transactionnel et dans le canal logistique. Le canal transactionnel concerne les aspects commerciaux et marketing visant à préparer l'échange (choix des produits, fixation des remises, conditions de retours etc.) alors que le canal logistique comprend des fonctions de prise en charge concrète de l'échange (préparation des commandes, transport, stockage, facturation, etc.). Surtout, les stratégies de résistance identifiées possèdent chacune un jeu de facteurs explicatifs qui leur est propre. Parmi les facteurs explicatifs, il y a évidemment le poids commercial, mesuré dans l'étude par le chiffre d'affaires réalisé. Or, si certaines stratégies sont intimement liées au poids commercial, d'autres restent relativement indépendantes de lui (voir Tableau 3). 
Tableau 3 - Les stratégies de résistance (Source : d'après Poirel, 2003)

\begin{tabular}{|c|l|l}
\hline $\begin{array}{c}\text { Conditions IType de } \\
\text { canal }\end{array}$ & \multicolumn{1}{|c|}{ Canal transactionnel } & \multicolumn{1}{c}{ Canal logistique } \\
\hline $\begin{array}{c}\text { Conditions liées à la } \\
\text { taille }\end{array}$ & $\begin{array}{l}\text { Confrontation transactionnelle: } \\
\text { - Centralisation des achats } \\
\text { - Arrangements extra- } \\
\text { contractuels }\end{array}$ & $\begin{array}{l}\text { Maîtrise des interfaces : } \\
\text { - Centralisation des } \\
\text { approvisionnements } \\
\text { - Arbitrage entre réseaux ce } \\
\text { distribution physique }\end{array}$ \\
\hline $\begin{array}{c}\text { Conditions } \\
\text { indépendantes de la } \\
\text { taille }\end{array}$ & $\begin{array}{l}\text { Sélectivité de l'offre : } \\
\text { - Refus de certaines mises en } \\
\text { place } \\
\text { - Réglage de l'office (envoi des } \\
\text { nouveautés) }\end{array}$ & $\begin{array}{l}\text { Rationalisation logistique : } \\
\text { - Optimisation du stock } \\
\text { - Gestion des flux entrants } \\
\text { sortants }\end{array}$ \\
\hline
\end{tabular}

La confrontation transactionnelle et la maîtrise des interfaces sont avant tout liées à des conditions de taille. Elles reflètent un certain poids commercial résultant soit d'un groupe de magasins intégrés appartenant à une même enseigne soit d'une association d'indépendants réalisant en commun un ensemble de services. Il s'agit de stratégies qui prennent appui sur une intégration du canal dans les domaines commercial et/ou logistique, via la création de centrale d'achats et/ ou de plates-formes de distribution. La confrontation transactionnelle vise alors à prendre le contrôle de la négociation commerciale par la contestation des conditions générales de vente des fournisseurs alors que la maîtrise des interfaces permet aux acteurs concernés de maîtriser, en totalité ou en partie, la gestion des flux en amont de leurs magasins de façon à ne pas se laisser imposer par les fournisseurs les paramètres de fonctionnement (envoi des nouveautés, gestion des retours etc.), de coûts (prix du transport, frais d'approches, etc.) et de services (délais de livraison, information sur les commandes, etc.).

La sélectivité de l'offre et la rationalisation logistique sont, en revanche, des stratégies de résistance pour lesquelles le poids commercial demeure secondaire. Interviennent des conditions plus qualitatives liées au climat des transactions, plutôt conflictuel ou plutôt confiant, ainsi qu'aux caractéristiques de l'environnement telles que l'intensité concurrentielle, la demande adressée au canal et la turbulence. Ces deux stratégies se conçoivent plus dans une logique de métier que dans une logique de taille. Le métier désigne toute profession dont on peut tirer des moyens d'existence ainsi que l'habileté que procure la pratique de la profession. Il met l'accent sur les compétences et les savoir-faire détenus par ceux qui travaillent dans l'entreprise alors que les notions de segment stratégique et de 
domaine d'activité se définissent en regard des marchés, des technologies et des produits. En adoptant le comportement de sélectivité de l'offre, les acteurs entendent garder la responsabilité pleine et entière de leurs assortiments sans ingérence des fournisseurs. Avec la rationalisation logistique, les acteurs s'impliquent dans une démarche d'optimisation du stock et de gestion interne des flux destinée à contrer les pratiques spéculatives des fournisseurs.

\section{2 - La logistique pour apprendre à résister}

Le deuxième enseignement de la recherche a désigné la logistique comme le domaine du canal de distribution par lequel les acteurs apprenaient à résister. Une analyse typologique a en effet montré que ceux-ci avaient des niveaux de résistance variables (faible, moyen et fort) et que ces niveaux étaient en relation avec des choix stratégiques particuliers. Si un premier profil s'est révélé faiblement résistant, ne cherchant pas à adopter une quelconque stratégie parmi les quatre évoquées précédemment («les indifférents»), un deuxième profil s'est montré plus pugnace vis-à-vis des fournisseurs en développant une résistance dans le domaine logistique («les sympathisants »). Profil le plus résistant de tous («les partisans »), le troisième s'est démarqué du précédent par des comportements transactionnels plus soutenus.

Figure 2 - De l'apprentissage à l'intensification de la résistance (source : Poirel, 2008)
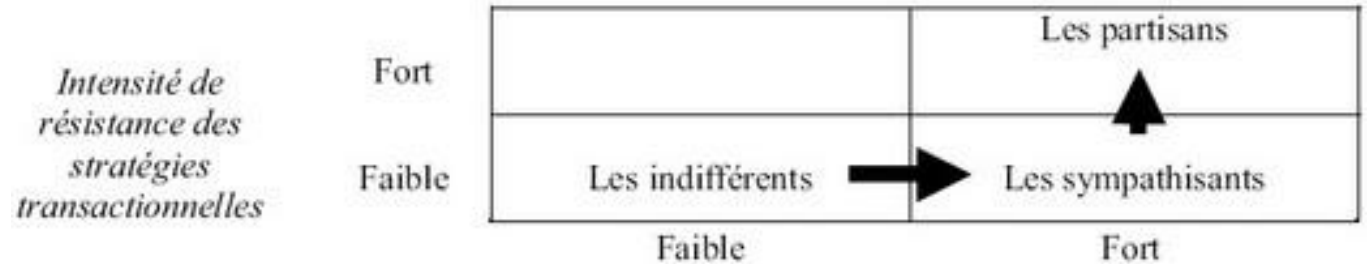

Intensité de résistance des stratégies logistiques

Tel que le montre la Figure 2, apparaît un parcours de «l'acteur résistant », qui démarre par une phase d'apprentissage où la résistance se traduit par l'adoption de stratégies de rationalisation logistique et de maîtrise des interfaces (passage des indifférents aux sympathisants) puis continue par une phase de développement qui voit s'intensifier la résistance par l'ajout de comportements transactionnels (passage des sympathisants aux partisans). Cette montée en puissance s'exprime dans un ensemble d'actions qui se cumulent et qui sont d'abord logistiques puis commerciales et marketing. C'est donc par la logistique 
que les acteurs du commerce signent leurs premiers actes de résistance, du moins dans le canal de distribution du livre en France.

\section{Conclusion}

Les axes de recherche développés par les chercheurs du CRET-LOG étoffent les théories sociopolitiques initiées par Stern (1969). Elles tendent à une meilleure explication du phénomène, en apportant des résultats sur les plans empiriques, théoriques et méthodologiques. Ainsi, Bonet (1999) utilise une méthodologie novatrice avec l'utilisation du logiciel Tropes pour mieux comprendre les comportements des acteurs du canal de distribution. Poirel (2003) cherche également à déchiffrer ces attitudes en utilisant l'approche stratégique. La notion de résistance qu'elle développe, lui permet d'affiner les effets du leadership dans le canal. Noireaux (2006) souligne les limites de l'exercice inconsidéré du pouvoir à travers la notion de reconnaissance du leadership. Son analyse longitudinale lui permet également d'identifier les formes d'exercice du pouvoir comme une régulation du canal dans un environnement hostile à sa survie. Enfin, Paché en collaboration avec Filser (Filser et Paché, 2008) intègre la dimension logistique à l'exercice du pouvoir, et revisite ainsi l'approche instrumentale proposée par Hunt et Nevin (1974).

L'ensemble des contributions présentées met également en évidence que l'analyse du canal de distribution est d'une grande richesse dès lors qu'on adjoint aux théories traditionnelles du canal d'autres approches, comme la logistique, la stratégie ou le marketing. Cette multidisciplinarité permet, en outre, de ne pas tomber dans un écueil : celui du pouvoir qui expliquerait tout! Telle était la thèse d'un article de March (1966) intitulé le pouvoir du pouvoir. La prise en compte d'éléments logistiques, stratégiques et marketing enrichit la compréhension des relations de pouvoir entre organisations et autorise une mise en perspective avec des problématiques proches mais distinctes, propres à la gestion des flux et à la complexité du jeu des acteurs. L'importance du pouvoir dans l'explication des phénomènes intra-organisationnels et surtout, en regard de la logique du canal, inter-organisationnels, se voit ainsi relativisée.

Enfin, les connivences de la distribution avec la logistique, la stratégie et le développement durable nous semblent constituer trois grands axes de recherche privilégiés par le CRET-LOG mais intéressant également l'ensemble de la communauté des chercheurs. En effet, en tant que 
maillon des chaînes logistiques multi-acteurs, la distribution est partie prenante du supply chain management, comme le montre un ouvrage collectif écrit conjointement par des chercheurs du CRET-LOG et du CERAG (Paché et Spalanzani, 2007). Quant aux liens qu'elle entretient avec la stratégie, il y en a un, celui de la dualité stratégique, qui occupe tout particulièrement les spécialistes qui sont en train d'élaborer un corpus théorique (PellegrinBouchet et Le Roy, 2008). Les stratégies de coopétition, qui mêlent à la fois coopération et compétition, partagent des problématiques communes avec le canal de distribution dans la mesure où les acteurs ne sont pas seulement impliqués dans des démarches collaboratives mais peuvent également emprunter la voie de la résistance. Enfin, un thème aux enjeux économiques et sociétaux majeurs, le développement durable, se prête tout particulièrement à la multidisciplinarité. C'est effectivement en associant les dimensions stratégiques et logistiques aux théories du canal, que Noireaux (2006) a pu révéler les apports du pouvoir et du leadership à l'analyse de la gestion des déchets industriels.

\section{Bibliographie}

- ADEME (2004), Les marchés relatifs aux déchets : situation 20022003 et perspectives pour 2004, ADEME Editions, Données et références, Paris.

- Anderson J.C. et Narus J.A. (1984) «A Model of The Distributors's Perspective of Distributor-Manufacturer Working Relationships », Journal of Marketing, Vol. 48, No. 4, p. 62-74.

- Baechler J. (1978), Le pouvoir pur, Calmann-Lévy, Coll. Archives des sciences sociales, Paris.

- Bonet D. (1999), Conflit et coopération dans le canal de distribution : l'analyse du discours des acteurs comme révélateur des comportements stratégiques, Thèse de doctorat en Sciences de Gestion, Université de la Méditerranée, Aix-Marseille II.

- Courpasson D. et Golsorkhi D. (coord.) (2008), «Variations autour d'une lecture dynamique du pouvoir dans les phénomènes stratégiques et organisationnels », Table ronde, XVII Conférence internationale de management stratégique, AIMS, Nice-Sophia Antipolis, 28-31 mai.

- Cox A. (2001), «Understanding buyer and supplier power: a framework for procurement and supply competence », The Journal of Supply Chain Management, Vol. 2, p.8-15, Spring.

- Crozier M. et Friedberg E. (1977), L'acteur et le système, Le Seuil, Paris.

Dahl R. (1971), Qui gouverne?, Armand Colin, Coll. Analyse Politique, Paris. 
- Dwyer F.R. (1980), «Channel-Member Satisfaction: Laboratory Insights », Journal of Retailing, Vol. 56, No. 2, p. 45-65.

- El-Ansary A.I. et Robicheau R.A. (1974), «A Theory of Channel Control: Revisited », Journal of Marketing, Vol. 38, p. 2-7.

- El-Ansary A.I. et Stern L.W. (1972) «Power Measurement in the Distribution Channel », Journal of Marketing Research, Vol. IX, No. 1, p.47-52, February.

- Emerson R.M. (1962), «Power dependence relations », American Sociological Review, No. 22, p.31-41.

- Etgar M. (1977), « Channel Environment and Channel Leadership », Journal of Marketing Research, Vol. XIV, p. 69-76.

- Etgar M. (1978), «Intrachannel Conflict and Use of Power », Journal of Marketing Research, May, Vol. XV, pp. 273-274.

- Filser M. (1989), Les canaux de distribution. Description, Analyse et Gestion, Vuibert Gestion, Paris.

- Filser M. (2000), «Les théories du canal de distribution : le dualisme des paradigmes », in : N. Fabbe-Costes, J. Colin et G. Paché, Faire de la recherche en logistique de distribution, Vuibert, Coll. FNEGE, Paris, p. 55-88.

- Filser M. et Paché G. (2008), «La dynamique des canaux de distribution: approches théoriques et ruptures stratégiques », Revue Française de Gestion, Vol. 34, No. 182, p.109-133.

- Frazier G.L. (1983), «On the Measurement of Interfirm Power in Channels of Distribution », Journal of Marketing Research, Vol. XX, p. 158-166, May.

- French J.R.P. et Raven B. (1959), «The Bases of Social Power», In : Cartwright, D. et Arbor, A., Studies in Social Power, University of Michigan Press, p. 150-167.

- Hunt S.D. et Nevin J.R. (1974), « Power in Channels of Distribution : Sources and Consequences », Journal of Marketing Research, Vol. XI, p. 186-193.

- Lapassouse C. (1989), Contribution à l'étude des facteurs d'évolution des canaux de distribution : l'exemple du négoce technique, Thèse de doctorat en Sciences de Gestion, Université Bordeaux I.

- Lapassouse C. (1991), «Comportement stratégique du distributeur industriel : différenciation et résistance », Recherche et applications en Marketing, Vol. VI, No. 2, p.99-110.

- Little R.W. (1970), «The Marketing Channel: Who should Lead This Extra-corporate Organisation? », Journal of Marketing, Vol. 34, p. 3138. 
- Lusch R.F. et Brown J.R. (1982), « Modified Model of Power in the Marketing Channel », Journal of Marketing Research, Vol. 19, p. 312323.

- Mallen B. (1964), «Conflict and Cooperation in Marketing Channels », In: G. Smith, Reflexions on Progress marketing, American Marketing Association, Chicago.

- March J.G. (1955), «An Introduction to the Theory and Measurement of Influence », American Political Science Review, No. 49, p.433-451, June.

- March J.G. (1966), «The power of power», in: D. Easton (edt), Varieties of Political Theory, Prentice-Hall, Englewood Cliffs, NJ, p. 39-70.

- Noireaux V. (2006), L'émergence du leadership reconnu dans le canal de distribution inversée: le cas des déchets industriels, Thèse de Doctorat en Sciences de Gestion, Université de la Méditerranée, AixMarseille II.

- Noireaux V. (2008), «Les leaders reconnus du canal de distribution inversée », 3ème Congrès RIODD, Lyon, 5 et 6 Juin.

- Paché G. (1999), «La logistique comme technologie de maîtrise des flux : une source de pouvoir aux mains des distributeurs », Colloque CNRIUT 99, Science et Technologie - Regards Croisés, Edition l'Harmattan, Paris.

Paché G. (2002), «L'évolution des relations logistiques entre industriels et détaillants : coopération ou simple coordination?», Gestion 2000, No. 1, p.109-124, Janvier-Février. Paché G. et Spalanzani A. (coord.) (2007), La gestion des chaînes logistiques multiacteurs: perspectives stratégiques, Presses Universitaires de Grenoble, Grenoble.

- Pellegrin-Boucher E. et Le Roy F. (2008), «Dynamique des stratégies de coopétition dans le secteur des TIC: le cas des ERP», XVII Conférence Internationale de Management Stratégique, AIMS, NiceSophia Antipolis, 28-31 mai.

- Poirel C. (2003), Les comportements de résistance dans le canal de distribution du livre en France. Approche sociopolitique et stratégique, Thèse de Doctorat en Sciences de Gestion, Université de la Méditerranée, Aix-Marseille II.

Poirel C. (2008), «La librairie en France: un management sous influence en quête de résistance », in : X. Greffe et N. Sonnac (coord), Culture Web, création, contenus, économie numérique, Dalloz, Paris, p.629-648.

- Porter M. (1982), Choix stratégique et concurrence, Economica, traduction française ; édition originale : 1980, Paris. 
- Porter M. (1986), L'avantage concurrentiel, Interéditions ; édition originale : 1985, Paris.

- Pras B. (1991), «Stratégies génériques et de résistance dans les canaux de distribution: commentaires et illustration », Recherche et Applications en Marketing, Vol. VI, No. 2, p. 111-123.

- Raven B.H. et Kruglanski A.W. (1970), «Conflict and power », in:: The Structure of conflict, Paul Swingle Edition, Academic Press, NewYork, p.69-109.

- Stern L.W. (1969), Distribution Channels: Behavioral Dimensions, Hougton Mifflin, Boston (MA).

- Stern L.W. et El-Ansary A.I. (1988), Marketing Channels, Prentice Hall, International Editions, New-Jersey.

- Stern L.W. et Heskett J. (1969), «Conflict Management in Interorganisational Relations: A Conceptual Framework », in Stern L.W., Distribution Channel: Behavioural dimensions, HoughtonMifflin, p. 288-305.

- Stern L.W. et Reve T. (1980), «Distribution Channels as a Political Economies: a Framework for Comparative Analysis », Journal of marketing, Vol. 44, p.52-64, Summer.

- Schul P.L., Pride W.N. and Little T.E. (1983), "The Impact of Channel Leadership Behavior on Intrachannel Conflict», Journal of Marketing, Vol. 47, No. 3, p. 21-34.

- Weber M. (1971), Economie et Société, Plon, traduction française, Coll. Recherches en sciences humaines, édition originale : 1922, Paris.

- Wilkinson I.F. (1979), «Power and Satisfaction in Channels of Distribution », Journal of Retailing, Vol. 55, $\mathrm{N}^{\circ}$ 2, p. 79-94. 